\title{
Fuzzy Risk-based Decision Method for Vehicular Ad Hoc Networks
}

\author{
Riaz Ahmed Shaikh \\ Computer Science Department, \\ King Abdulaziz University, \\ Jeddah, Saudi Arabia
}

\begin{abstract}
A vehicular ad hoc network (VANET) is an emerging technology that has the potential to improve road safety and traveler comfort. In VANETs, mobile vehicles communicate with each other for the purpose of sharing various kinds of information. This information is very useful for preventing road accidents and traffic jams. On Contrary, bogus and inaccurate information may cause undesirable things, such as automobile fatalities and traffic congestion. Therefore, it is highly beneficial to consider risk before vehicle takes any decision based on the received information from the surrounding vehicles. To overcome these issues, we propose a new risk-based decision method for vehicular ad hoc networks. It determines a risk-based the three key elements: 1) application type and sensitivity level, 2) vehicle context and 3) driver's attitude. This paper also provides theoretical analysis and evaluation of the proposed method, and it also discusses the applications of the proposed model.
\end{abstract}

Keywords-Ad hoc networks; Decision methods; Risk management; Trust management; Vehicular Networks

\section{INTRODUCTION}

In vehicular ad hoc networks (VANET), vehicles and roadside units (RSUs) communicate with each other by sharing various types of information, such as safety-related warning messages. This collaborative communication is very helpful in avoiding road accidents and traffic congestion. However, accuracy and reliability of the received information need to be evaluated first before vehicle takes any decision, such as change of lane or road, etc. For this purpose, various researchers [1-7] recommend the use of trust management schemes in VANETs.

Many trust management methodologies [1-7] exist in the literature that mainly focuses on evaluating the trustworthiness of the received data in VANETs. However, less attention is given to the decision-making. From VANET's perspective, decision making is very critical, because consequences of any wrong decision could be disastrous, such as fatal road accidents. In most of the trust management approaches, the decision logic is straight forward. For example, a message having the maximum trust value is accepted [4], or any message that is coming from the vehicle whose reputation is good is accepted [5]. Some researchers used simple majority or weighted majority voting techniques [6] for the decision making. However, most of the existing works do not consider risk in the decision making in VANETs.
A risk can be defined as a level of uncertainty resulting from the potential for a negative outcome. In the VANETs, it can be viewed from an application sensitivity level. In general, VANET applications can broadly be categorized into three types: 1) safety applications, 2) traffic efficiency applications, and 3) infotainment applications. A message that is generated by a safety application may have higher sensitivity level than a message which is generated by a traffic efficiency application. Similarly, messages that are generated by a traffic efficiency application have higher sensitivity level than an infotainment application messages. So, the application which has high sensitivity level imposes high risk. Furthermore, two or more conflicting messages belonging to the same application may impose various risks depending upon its consequences. Other than the application type, risk can also be evaluated by considering other contextual attributes that can affect the physical and mental condition of the driver, etc. [8].

Consider two messages $m 1$ ("Road $X$ is icy at intersection $X-Y$ ") and $m 2$ ("Road $X$ is dry at intersection $X-Y$ ") are received by the vehicle from the surrounding vehicles. With the help of any existing trust management scheme, assume that the trust value of $m 1$ is 0.4 that is higher than the trust value of the message $m 2$ (e.g., 0.3). So, $m 1$ is considered more trustworthy than $m 2$. However, from the sensitivity level perspective of the application, trust value of $m 1$ is less than the acceptable threshold value (e.g., 0.5). Now, the problem is: How much risk we are willing to take to accept some relatively trustworthy message? Or in other words: "If the trust level of the message is low with respect to the sensitivity level of the application, should we accept that message?" This is the critical and challenging problem, which is commonly overlooked during the decision-making process in vehicular networks.

In this work, we have proposed a new fuzzy risk-based decision method for vehicular ad hoc networks. In this method, the risk is estimated based on three key factors: 1) application type and it's sensitivity level, 2) vehicle context and 3) driver's attitude. The use of these three dimensions in the risk estimation makes our proposal unique. Furthermore, it also provides a better degree of completeness. We have provided solutions to measure risk in both qualitative and quantitative manner, which will increase its applicability in various scenarios. Additionally, we have also provided a data set that can be used to create benchmarks for the comparison purposes.

The rest of the paper is organized as follows. Section 2 
discusses the related work. Section 3 describes the proposed fuzzy risk-based decision method. Section 4 contains analysis and evaluation of the proposed method. Section 5 depicts the applications of the proposed method. Finally, section 6 concludes the paper and outline future work directions.

\section{RELATED WORK}

Decision making is usually described as a mental process of selecting the best one from judging multiple options or alternatives [9]. The decision-making process commonly involves the following five activities [9][10][11]:

- decision problem identification;

- relevant information collection and verification;

- identification of the decision substitutes;

- foresee the consequences of decisions;

- decision making;

In this work, we are mainly focusing on the decisionmaking process of trust models that are primarily used for making reliable decisions. However, existing trust models do not consider the consequence of a wrong decision, which we are referring here to a risk. Description about the decisionmaking process of existing state-of-the-art trust models is given below.

Shaikh and Alzahrani [1] have proposed a trust management scheme for VANETs. The unique thing about their method is that it evaluates trust in an environment where identities of nodes are not known. It works in three phases. Firstly, each receiver node will measure a confidence value for each received message that is based on four parameters: 1) Time closeness, 2) Location closeness, 3) Time verification and 4) Location verification. Secondly, it calculates the trust value for each unique message. Finally, it takes the decision. The decision process is comprised of two steps: First, it selects the message which has the highest trust value. Second, if the selected message's trust value is exceeding the least acceptable threshold value, then the message will be accepted. Otherwise, it will be discarded.

Cohen's et al. [2] proposed trust method first measured the confidence value for each received report that is based on various factors, such as, history, time, location and role. After that, the method will take the decision. For this purpose, they adopted majority-based trust model. If the majority confidence is greater than the acceptable threshold and the number of reports is greater than the pre-defined variable ' $n$,' then the method will follow the advice in the report. Else, it will follow the advice of the report with the highest confidence value.

Mármol and Pérez [5] have proposed a trust and reputation method for vehicular networks. In their model, whenever a node receives a message, it first assesses the reputation of the sender. It is measured based on three factors: 1) history, 2) recommendation from the neighbor vehicle, and 3) recommendation from the central authority via roadside units (RSUs). Based on the reputation score, a sender is classified into one of the three trust levels: 1) untrusted, 2) +/- trust, and 3) trusted. These levels are represented as fuzzy sets. After that, receiver node takes the decision. If the sender node belongs to first set (not trusted), then the message is rejected. If it belongs to a second set (+/- trust), then the message is considered reliable with tunable probability. However, it will not be broadcasted or forwarded to any other node. If the node belongs to a third set (trusted), then the message is accepted.

Wei and Chen [7] have proposed an adaptive decisionmaking method for improving the efficiency of the trust management system of VANETs. The objective of their work is to make the quick accurate decision. The decision-making process will trigger in two cases: 1) when the number of messages received is more than the specific threshold $\left(\mathrm{M}_{\max }^{\mathrm{rsu}}\right)$, or 2) when the time delay is exceeding the specific threshold $\left(\mathrm{t}_{\text {wait }}^{\text {rsu }}\right)$. Once the decision-making process triggered, it will take the decision by looking at the overall trust value of the event. If the trust value is greater than the given threshold value $\left(\mathrm{T}_{\text {thld }}\right)$, then the positive decision will be taken. Else, the method will consider that message as untrustworthy one.

U. F. Minhas et al. [12] have proposed a multi-faceted trust modeling framework for VANETs. In that framework, authors have incorporated the concepts of role-based trust, experience based trust, and majority-based trust. Among these three aspects, first two are used to select the advisors. Based on the recommendation of the selected advisors, the system takes the decision by adopting majority voting technique.

Tajeddine et al. [13] have proposed a framework which focuses on trust, reputation, and privacy-preserving trust system. In their paper, authors have shown that group decision and trust calculation of the received message are useful to increase the security in the VANET. Through this framework, the privacy of the users is respected using group decision organized by group management. Also, it provides security through trust and reputation. From this model, many attributes of security affecting trust calculation can be established using the group decision.

Huang et al. [14] have shown that simple voting for decision-making, leads to oversampling and gives wrong results using their proposed research. From this, they claimed that decision making based on trust management in mobile ad hoc networks is not suitable to VANET.

According to [15], authors have mentioned that intelligent transport system is maintainable through the appropriate signal quality which improves the decision-making ability in the VANET. Through the appropriate channel, decision-making, and solutions based on signal interference, strength and quality can be improved. The TrafficInfo algorithm is used to minimize the risk based on collisions and adjusts the number of dissemination reports included in the transmission. Risks are reduced through the adaptive control of transmission size [16]. The quality of the route which influences with VANET depended on the multi-metric routing decisions is proposed in [17].

Yang et al. [18] have proposed a dynamic three-layer reputation evidence decision and management mechanism, which combine with Dempster-Shafer evidence integration mechanism to distinguish selfish nodes and the risks which are suspect collusion vehicle nodes. A hierarchical Reputation 
Evidence Decision System (REDS) based on the DempsterShafer evidence theory is defined to establish the reputation management which increases the reputation accuracy in the VANET. Through this theory, the degree of trust can be calculated.

Fernandes et al. [19] have proposed a decentralized reputation system for vehicular networks (RS4VANET). The objective of this system is to guarantee the proper operation of a data dissemination application in the presence of malicious nodes. The proposed system follows an optimistic approach and uses various techniques to assess the trustworthiness of vehicles. For the decision-making, authors have adopted the concept of voting schemes.

\section{PROPOSED SOLUTION}

As discussed earlier, risk can be derived from the application sensitivity level. Other than the application, different other factors could also be used in risk estimation as shown in Fig. 1. Our proposed risk assessment framework is composed of three factors: 1) Application type, 2) Vehicle context and 3) Driver's attitude.

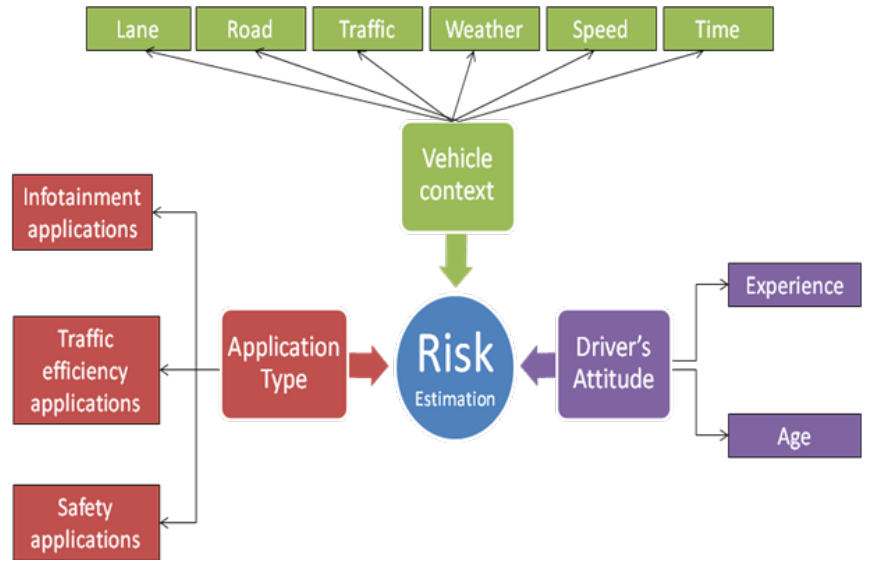

Fig. 1. Risk Estimation factors for VANET

The USA National Institute of Standards and Technology (NIST) [20] has defined risk as follows:

$$
\text { Risk }=\text { Threat likelihood } \times \text { Impact }
$$

At a high-level, we adopt the same definition. In our context, we determine the threat likelihood based on the vehicle context as well as driver's attitude and the impact based on the application sensitivity level.

\section{A. Impact}

The impact can be derived from a sensitivity value of the application. The higher the sensitivity value of the application, the higher the impact will be. There are two possible cases in which the impact can be derived: when the sensitivity value of an application is available or when it is unavailable. known

Case 1: when the sensitivity value of an application is

Let us assume that the sensitivity value $[0,10]$ is assigned to each application by the vendor. Assigning sensitivity value $\left(S_{v}\right)$ from a specific range to an application is a subjective matter. Therefore, we decided to use fuzzy logic to derive an impact level. This process is composed of two steps. In the first step, we determine the level of impact based on the sensitivity value, and in the second step, we determine the impact value in a quantitative manner by applying a mapping function.

Let us assume that there are three levels of impact: Low, Medium, and High, which can be considered as three sets. As compared to the classical set theory, the operations on fuzzy sets are based on the membership functions, which are typically linear and often take the shape of a triangle, trapezoid, or L [21]. The objective of the membership function is to determine the degree of truth that the element (i.e. sensitivity value) belongs to the particular set (i.e. low, medium or high).

In this work, we used Trapezoidal-shaped membership function. The reason for using this function is that it increases the flexibility, and it also allows an 'interval of values' that maximized the individual membership functions [22]. For example, Fig. 2 shows the trapezoid shape for low, medium and high fuzzy sets for sensitivity values. Mathematically, the membership functions that are shown in this figure are specified as below.

$$
\begin{gathered}
\mathrm{f}^{\text {Low }}\left(\mathrm{S}_{\mathrm{v}}\right)=\max \left(\min \left(\mathrm{S}_{\mathrm{v}}+1,1,3-\mathrm{S}_{\mathrm{v}}\right), 0\right) \\
\mathrm{f}^{\text {Medium }}\left(\mathrm{S}_{\mathrm{v}}\right)=\max \left(\min \left(\mathrm{S}_{\mathrm{v}}-2,1,7-\mathrm{S}_{\mathrm{v}}\right), 0\right) \\
\mathrm{f}^{\text {High }}\left(\mathrm{S}_{\mathrm{v}}\right)=\max \left(\min \left(\mathrm{S}_{\mathrm{v}}-6,1,11-\mathrm{S}_{\mathrm{v}}\right), 0\right)
\end{gathered}
$$

Note that the key feature of the fuzzy set is that there is no hard rule how boundaries of membership functions are defined. These can be set by taking input from the experts.

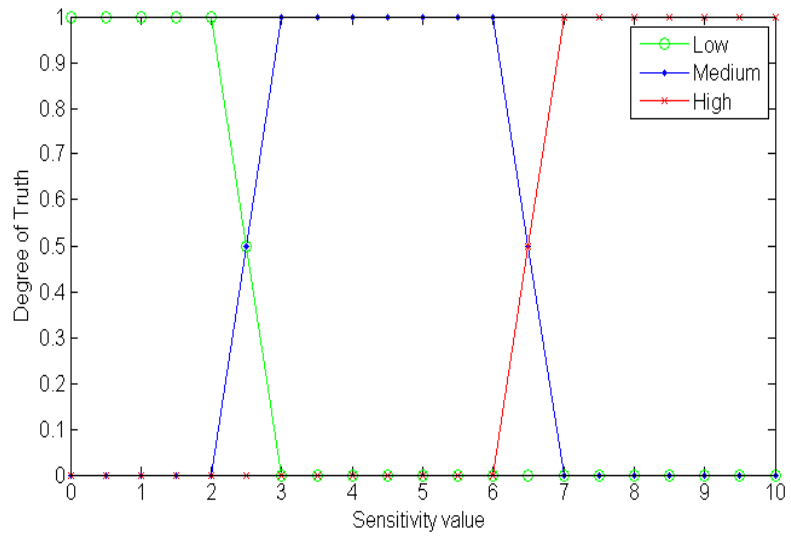

Fig. 2. Impact determination example using fuzzy set

After determining the impact level, we applied the following function to derive an impact value in a quantitative manner.

$$
\text { Impact }\left(S_{v}\right)=\left\{\begin{array}{ccl}
0 & \mathrm{f}^{\text {Low }}\left(\mathrm{S}_{\mathrm{v}}\right)==1 & \text { //low impact } \\
1 & \mathrm{f}^{\text {Medium }}\left(\mathrm{S}_{\mathrm{v}}\right)==1 & / / \text { medium impact } \\
2 & \mathrm{f}^{\text {High }}\left(\mathrm{S}_{\mathrm{v}}\right)=1 & / / \text { high impact } \\
2 & \text { else } & / / \text { default value }
\end{array}\right.
$$

In this function, we mapped the low, medium and high 'impact levels' with 'impact values' zero, one, and two respectively. However, other values could also be used by keeping the following condition intact. 


$$
\mathrm{v}(\text { Low })<v(\text { Medium })<v(\text { High })
$$

Where $v$ represent the value. Note that in this function (Eq. 5), the default value is same as the highest value. The reason for adopting this pessimistic approach is to avoid any undesirable things that may occur in the vehicular networks.

Case 2: when the sensitivity value of an application is unknown

In the absence of sensitivity value, the impact can be derived from the application type. As discussed earlier, there are three types of applications: infotainment applications, traffic efficiency applications, and safety applications. Based on the application type, we can determine the sensitivity level, and that will be used to determine impact.

The sensitivity level of infotainment applications, traffic efficiency applications, and safety applications can be assigned as low, medium and high respectively as shown in Fig. 3.

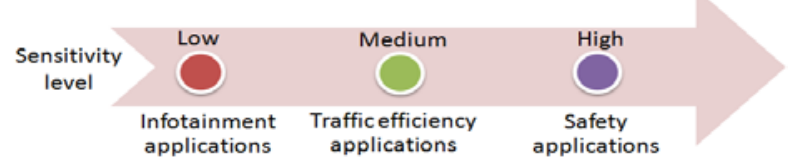

Fig. 3. Sensitivity level of VANET's applications

To estimate impact in a quantitative manner, we first assign numbers to the sensitivity levels of the application in the following manner.

$$
\mathrm{S}_{\mathrm{l}}=\{\text { Low }=0, \text { Medium }=1, \text { High }=2\}
$$

Note that different labels and numbers could also be used. Formally, we can define the relationship between the impact and $S_{1}$ in the following manner.

$$
\text { Impact } \propto S_{1}
$$

The above relationship shows that the impact is directly proportional to the sensitivity level of the application $\left(\mathrm{S}_{1}\right)$.

\section{B. Threat Likelihood}

Threat Likelihood can be measured based on the vehicle context $\left(\mathrm{V}_{\text {context }}\right)$ and driver's attitude $\left(\mathrm{D}_{\text {attitude }}\right)$.

$$
\begin{gathered}
\text { Threat likelihood }=\beta_{1} \mathrm{~V}_{\text {context }}+\beta_{2} \mathrm{D}_{\text {attitude }} ; \\
\beta_{1}+\beta_{2}=1
\end{gathered}
$$

Where $\beta_{1}$ and $\beta_{2}$ represent the weight values for $V_{\text {context }}$ and $\mathrm{D}_{\text {attitude }}$ parameters respectively.

\section{1) Vehicle context}

Vehicle context can be determined based on the following factors:

a) Lane $\left(L_{e}\right)$, e.g., straight, curve, winding, Uphill, Downhill, Intersection, Corner.

b) $\operatorname{Road}\left(R_{d}\right)$, e.g., dry, wet, icy.

c) Traffic $\left(T_{c}\right)$, e.g., a car in front, car on left, car on right, a car in rear. windy. e) Speed $\left(S_{d}\right)$, e.g., accelerating, decelerating.

\begin{tabular}{|c|c|c|c|c|c|}
\hline $\begin{array}{r}\text { Lane } \\
\left(\boldsymbol{L}_{e}\right) \\
\end{array}$ & $\begin{array}{c}\text { Weather } \\
\left(\boldsymbol{W}_{r}\right) \\
\end{array}$ & $\begin{array}{l}\text { Time } \\
\left(T_{e}\right) \\
\end{array}$ & $\begin{array}{c}\text { Traffic } \\
\left(\boldsymbol{T}_{c}\right) \\
\end{array}$ & $\begin{array}{r}\text { Road } \\
\left(\boldsymbol{R}_{d}\right) \\
\end{array}$ & $\begin{array}{c}\text { Speed } \\
\left(\boldsymbol{S}_{d}\right) \\
\end{array}$ \\
\hline $\begin{array}{l}\text { Straight } \\
\text { (L) }\end{array}$ & $\begin{array}{l}\text { Clear } \\
\text { (L) }\end{array}$ & $\begin{array}{l}\text { Day } \\
\text { (L) }\end{array}$ & $\begin{array}{c}\text { Car in front } \\
(\mathrm{H})\end{array}$ & $\begin{array}{l}\text { Dry } \\
\text { (L) }\end{array}$ & $\begin{array}{c}\text { Accelerating } \\
(\mathrm{H})\end{array}$ \\
\hline $\begin{array}{l}\text { Curve } \\
(\mathrm{H})\end{array}$ & $\begin{array}{l}\text { Raining } \\
\text { (L) }\end{array}$ & $\begin{array}{l}\text { Night } \\
(\mathrm{H})\end{array}$ & $\begin{array}{l}\text { Car on left } \\
\text { (M) }\end{array}$ & $\begin{array}{l}\text { Wet } \\
(\mathrm{M})\end{array}$ & $\begin{array}{l}\text { Decelerating } \\
\text { (L) }\end{array}$ \\
\hline $\begin{array}{l}\text { Winding } \\
\text { (M) }\end{array}$ & $\begin{array}{c}\text { Snowing } \\
(\mathrm{H})\end{array}$ & $\begin{array}{l}\text { Dusk } \\
\text { (M) }\end{array}$ & $\begin{array}{l}\text { Car on right } \\
\text { (M) }\end{array}$ & $\begin{array}{l}\text { Icy } \\
(\mathrm{H})\end{array}$ & \\
\hline $\begin{array}{l}\text { Uphill } \\
\text { (M) }\end{array}$ & $\begin{array}{c}\text { Foggy } \\
(\mathrm{H})\end{array}$ & $\begin{array}{c}\text { Dawn } \\
\text { (M) }\end{array}$ & $\begin{array}{c}\text { Car in rear } \\
(\mathrm{H})\end{array}$ & & \\
\hline $\begin{array}{l}\text { Downhill } \\
\text { (M) }\end{array}$ & $\begin{array}{l}\text { Windy } \\
\text { (M) }\end{array}$ & & & & \\
\hline $\begin{array}{c}\text { Intersectio } \\
n \\
(\mathrm{H})\end{array}$ & & & & \multirow{2}{*}{\multicolumn{2}{|c|}{$\begin{array}{c}\mathrm{L}=\text { Low Risk } \\
\mathrm{M}=\text { Medium Risk } \\
\mathrm{H}=\text { High Risk }\end{array}$}} \\
\hline $\begin{array}{l}\text { Corner } \\
(\mathrm{H})\end{array}$ & & & & & \\
\hline
\end{tabular}

f) Time $\left(T_{e}\right)$, e.g., day, night, dusk, dawn.

Table 1 shows the fuzzy risk levels for the values of the above-mentioned factors.

TABLE I. Vehicle Context Parameters

Based on these factors of vehicle context, we can determine the risk in the following manner:

$$
\begin{aligned}
& \mathrm{V}_{\text {context }}=\max \left(\mathrm{w}_{1} \mathrm{~L}_{\mathrm{e}}, \mathrm{w}_{2} \mathrm{R}_{\mathrm{d}}, \mathrm{w}_{3} \mathrm{~T}_{\mathrm{c}}, \mathrm{w}_{4} \mathrm{~W}_{\mathrm{r}}, \mathrm{w}_{5} \mathrm{~S}_{\mathrm{d}}, \mathrm{w}_{6} \mathrm{~T}_{\mathrm{e}}\right) ; \\
& \sum_{\mathrm{i}=1}^{6} \mathrm{w}_{\mathrm{i}}=1
\end{aligned}
$$

Where $w_{i}$ represents the weight value for the specific parameter.

\section{2) Driver's attitude}

To derive driver's attitude, we use two factors in our model: 1) Age (A) and 3) Experience (E).

$$
D_{\text {attitude }}=\max \left(\alpha_{1} A, \alpha_{2} E\right)
$$

Age and experience play a vital role in the driving performance. Younger drivers and old drivers are mostly involved in an accident more than middle age drivers. There is no consensus regarding higher threshold age for young drivers and lower threshold age for old drivers. As stated in [23], 65 is a most commonly accepted age for defining the older driver and it is an age where accident rate begins to increase. Upper limit defined for the younger drivers is 25 [24]. We can determine the risk based on the age factor in the following way.

$$
A=\left\{\begin{array}{cl}
\text { Low } & \text { if } 35<\text { Age } \leq 75 \\
\text { Medium } & \text { if } 25<\text { Age } \leq 35 \\
\text { High } & \text { if } \text { Age } \leq 25 \text { OR Age } \geq 75
\end{array}\right.
$$

We can determine the risk based on the experience factor in the following way.

- Low, when the driver has experience of more than 30 years.

- Medium, when the driver has experience of more than ten years and less than 30 years.

- High, when the driver has experience of fewer than ten years. 
Formally, we can define these rules in the following way.

$$
\mathrm{E}=\left\{\begin{array}{cl}
\text { Low } & \text { if Experience } \geq 30 \\
\text { Medium } & \text { if } 10<\text { Experience }<30 \\
\text { High } & \text { if Experience } \leq 10
\end{array}\right.
$$

\section{Qualitative Risk estimation}

As mentioned in Equation 1, the risk is the product of threat likelihood and impact. This formulation gives us the quantitative value of the risk. In some scenarios, we may want to determine risk in a qualitative manner. In such situations, we need some mapping function that can be used to convert quantitative values in a qualitative manner.

Let us assume that the maximum value of a risk is $\mathrm{n}$ and the number of risk levels is $\mathrm{k}$. Then we can use the following mapping function.

$$
\begin{aligned}
& \text { Risk level } \\
& =\left\{\begin{array}{cc}
\text { level }-\mathrm{k} & (\mathrm{k}-1) \mathrm{n} / \mathrm{k}<\text { Risk } \leq n \\
\vdots & \vdots \\
\text { level }-2 & \mathrm{n} / \mathrm{k}<\text { Ris } \leq \leq 2 \mathrm{n} / \mathrm{k} \\
\text { level }-1 & 0 \leq \text { Risk } \leq \mathrm{n} / \mathrm{k}
\end{array}\right.
\end{aligned}
$$

Example: Assume that the maximum risk value is two, and there are three risk levels (low, medium and high). In this scenario, the above-mentioned mapping function will be simplified in the following way.

Risk level $=\left\{\begin{array}{cc}\text { High } & 2 \times 2 / 3<\text { Risk } \leq 2 \\ \text { Medium } & 2 / 3<\text { Risk } \leq 2 \times 2 / 3 \\ \text { Low } & 0 \leq \text { Risk } \leq 2 / 3\end{array}\right.$

\section{THEORETICAL ANALYSIS AND EVALUATION}

As mentioned earlier, we have determined risk based on the product of impact and threat likelihood. We derived the impact based on the sensitivity level of the application and threat likelihood based derived from vehicle context and driver's attitude. To find out the impact of various parameters on the cumulative risk, we developed a small program which generates 100 different scenarios as shown in Table 2 (See Appendix).
For each scenario, first, we derived the impact from the sensitivity level [1-10] by implementing Equations 2 to 5. The Fig. 4 shows the impact for each scenario. One can see that the impact value fluctuates between 0 and 2, where 0 means low and 2 means high impact.

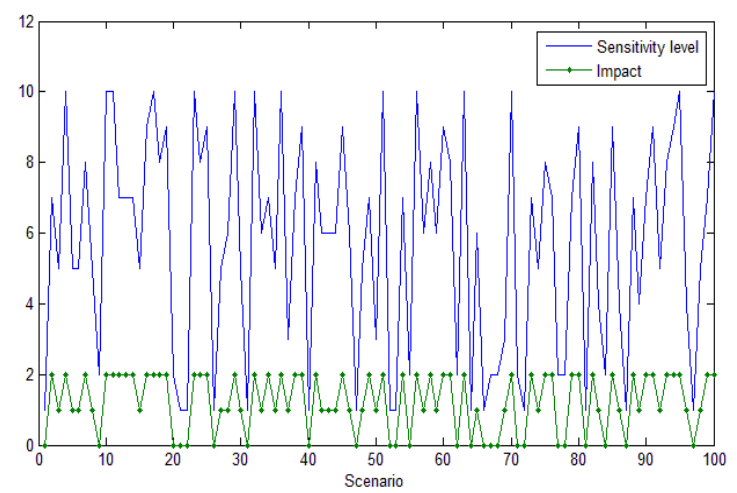

Fig. 4. Impact Analysis

To derive the threat likelihood, first, we calculate the vehicle context by implementing Equation 10 and driver's attitude by implementing Equation 11. Vehicle context is shown in the form of area chart (as shown in Fig. 5) which illustrate cumulative totals using number over each scenario. Note that, in this analysis; as indicated in the caption of Fig. 5, the weight value assign to each parameter are not same.

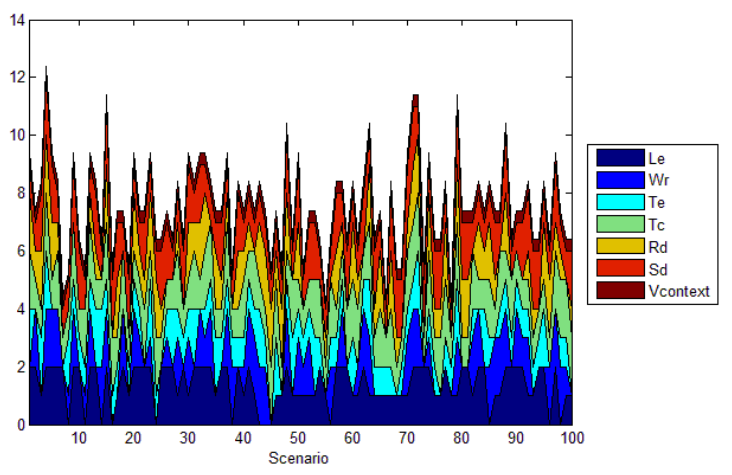

Fig. 5. Vehicle Context Analysis $\left(\mathbf{w}_{\mathbf{1}}=\mathbf{w}_{\mathbf{4}}=\mathbf{w}_{\mathbf{5}}=\mathbf{w}_{\mathbf{6}}=\mathbf{0 . 2} ; \mathbf{w}_{2}=\mathbf{w}_{\mathbf{3}}=\right.$ 0. 1) 
The impact of Age and Experience on driver's attitude is shown in Fig. 6 that is derived by applying equations 11 to 13 .

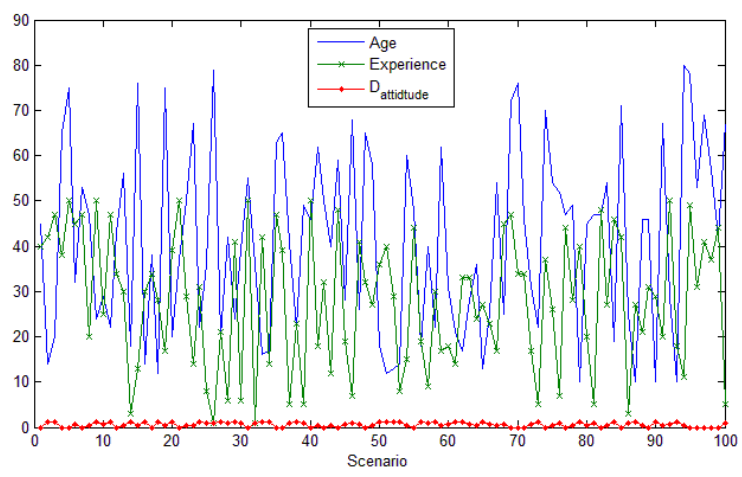

Fig. 6. Driver's attitude Analysis $\left(\boldsymbol{\alpha}_{\mathbf{1}}=\mathbf{0} . \mathbf{6}, \boldsymbol{\alpha}_{\mathbf{2}}=\mathbf{0 . 4}\right)$

Threat likelihood is derived by implementing Equation 9 . Fig. 7 shows the impact of vehicle context and driver's attitude on the threat likelihood. For the demonstration purposes, we have assigned a higher weight to the vehicle context $\left(\beta_{1}=0.6\right)$ as compared to the driver's attribute $\left(\beta_{2}=0.4\right)$.

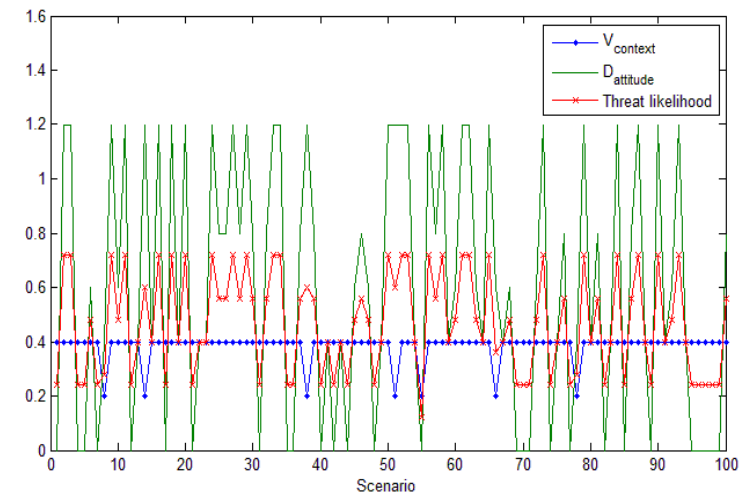

Fig. 7. Threat Likelihood Analysis $\left(\boldsymbol{\beta}_{\mathbf{1}}=\mathbf{0 . 6}, \boldsymbol{\beta}_{\mathbf{2}}=\mathbf{0 . 4}\right)$

Risk determination for each scenario based on threat likelihood and impact is shown in Fig. 8.

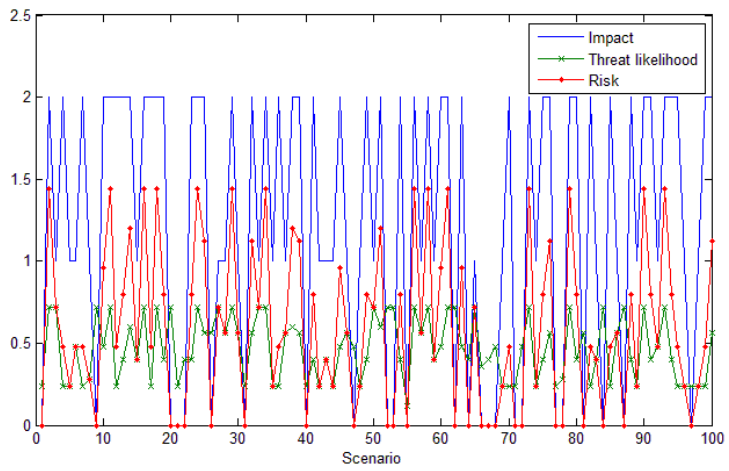

Fig. 8. Risk estimation based on Impact \& Threat likelihood
To determine the risk in a qualitative manner, we applied the Equation 15. Results are shown in Fig. 9a. According to the results, risk values of 14, 28 and 58 scenarios are classified as high, medium and low respectively as indicated in Fig. $9 \mathrm{~b}$.

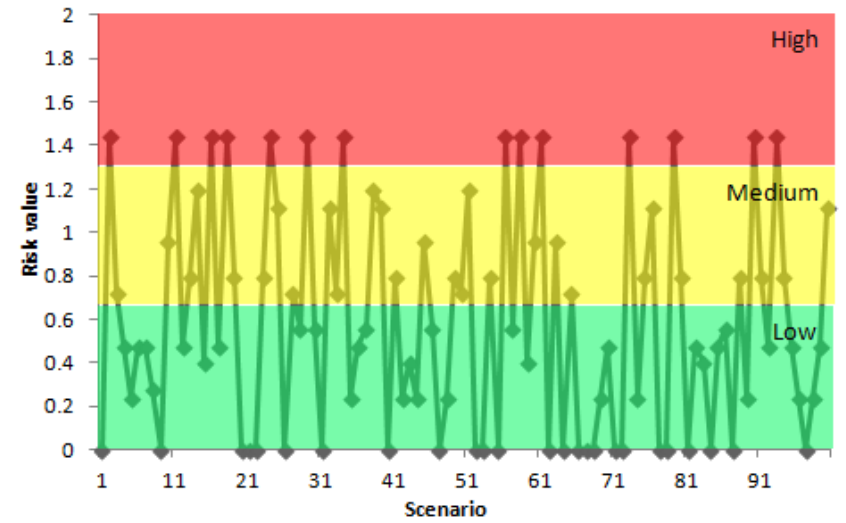

(a)

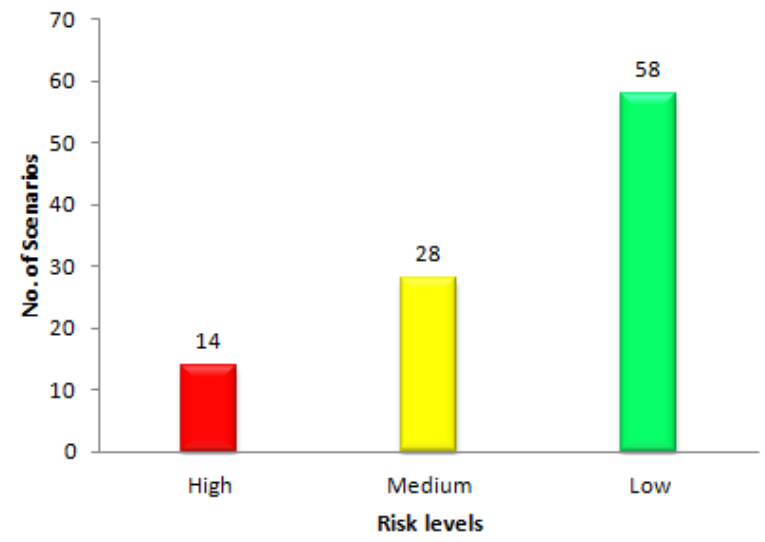

(b)

Fig. 9. Qualitative Risk estimation, (a) The risk value for each scenario, (b) Scenario classification w.r.t. risk level

\section{ApPlication OF the Proposed Method}

Our proposed method can assist existing trust management schemes of VANETS to make reliable decisions. For example, most of the trust management schemes [1, 2, 7], first measure the trust value based on various factors and then compare it with some pre-defined threshold value. If the trust value is greater than the threshold value, then the message is accepted. Otherwise, it will be discarded. Most of the researchers do not define how to calculate this pre-defined threshold value. The risk value that we are calculating in this work could be used as a threshold value. First order diagram of this concept is shown in Fig 10. 


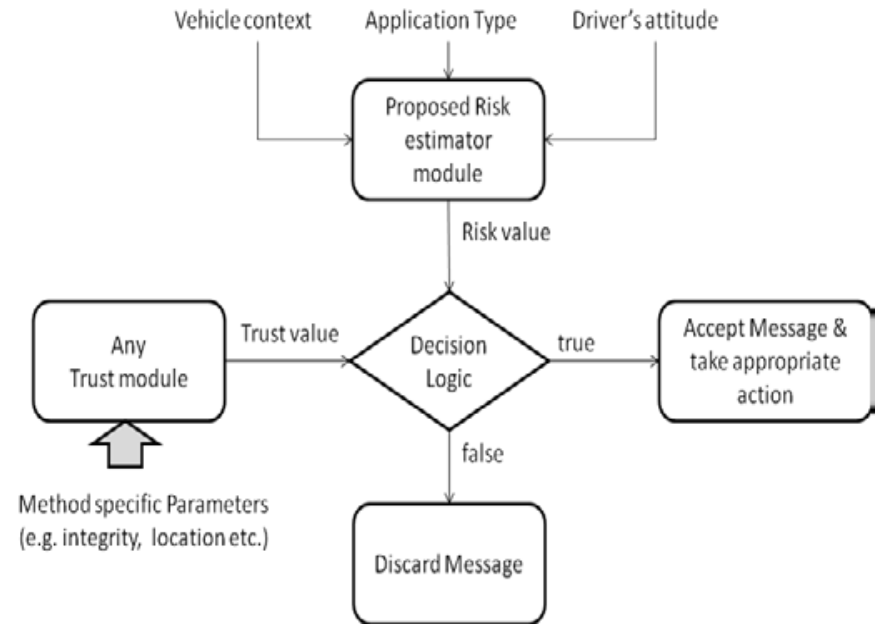

Fig. 10. Application of the Risk method

Another interesting application of our proposed model is its use in autonomous vehicles. Recently, many organizations like Google have developed prototypes of driverless cars. Google Inc. said their self-driving car will be ready by 2020 [25]. Driverless vehicles need similar techniques as standard programs which provide the decision dynamically. In this case, vehicle context and application type will be useful for direct applications because weather conditions and road situation are continuously changing with many parameters considered in our model. Although driver's attitude may not involve directly in the driverless vehicles, we can derive threat likelihood directly from the vehicle context. In the near future, we will see both regular and driverless vehicles on the roads. So, risk-based decision method will play a vital role. For example, when different drivers are driving their vehicles close to the driverless vehicles, this feature may be useful for providing safety and comfort to the passengers.

\section{CONCLUSION AND FUTURE WORK}

Traditional decision methods are not suitable for vehicular ad hoc networks. Due to its sensitivity, a risk must be considered in the decision-making process. Therefore, in this work, we proposed a new fuzzy risk-based decision method for vehicular ad hoc networks. In this method, we derived the risk based on the three key factors: 1) application type and sensitivity level, 2) vehicle context, and 3) driver's attitude. We measured risk both in qualitative and quantitative manner. Since benchmarks are not available for this domain, therefore, we have provided detailed data set (See Appendix). Based on this data set, we have conducted a theoretical analysis and evaluation of the proposed method. We hope that this will be helpful for the researchers in this field to come up with better solutions and make a comparison. In future, we would like to extend our model by adding more parameters like gender, traffic density, speed limits etc.

\section{REFERENCES}

[1] Shaikh, R. A., \& Alzahrani, A. S., "Intrusion-aware trust model for vehicular ad hoc networks", Security and Communication Networks, vol. 7 (11), pp. 1652-1669, 2014

[2] Cohen, R., Zhang, J., Finnson, J., Tran, T., \& Minhas, U. F., “A TrustBased Framework for Vehicular Travel with Non-Binary Reports and Its
Validation via an Extensive Simulation Testbed”, Journal of Trust Management, vol. 1(1), 2014.

[3] Huang, D., Hong, X., \& Gerla, M., "Situation-aware trust architecture for vehicular networks", Communications Magazine, IEEE, vol. 48(11), pp. 128-135, 2010.

[4] Zhang, J., "Trust management for vanets: Challenges, desired properties and future directions”, International Journal of Distributed Systems and Technologies (IJDST), vol. 3(1), pp. 48-62, 2012.

[5] Gómez Mármol, F., \& Martínez Pérez, G., “TRIP, a trust and reputation infrastructure-based proposal for vehicular ad hoc networks”, Journal of Network and Computer Applications, vol. 35(3), pp. 934-941, 2012.

[6] Ostermaier, B., Dotzer, F., \& Strassberger, M., "Enhancing the security of local danger warnings in vanets-a simulative analysis of voting schemes”, In the 2nd International Conference on Availability, Reliability and Security (ARES 2007), Vienna, Austria, April 2007, pp. 422-431.

[7] Wei, Y. C., \& Chen, Y. M., “Adaptive Decision Making for Improving Trust Establishment in VANET", 16th Asia-Pacific Network Operation and Management Symposium (APNOMS), Taiwan, Sep 2014, pp. 1-4

[8] Vijey T., Alzahrani, A., \& Qureshi, M. S., "Risk prediction system based on MIMO system for vehicle users", Life science Journal, vol. 10(4), pp. 3055-3061, 2013.

[9] Bohanec, Marko. "Decision making: A computer-science and information-technology viewpoint." Interdisciplinary Description of Complex Systems, vol. 7( 2), pp. 22-37, 2009.

[10] Skinner, D.C, "Introduction to Decision Analysis", Probabilistic Publishing, Gainesville, 3rd Edition, 2009

[11] Clemen, R.T, "Making Hard Decisions: An Introduction to Decision Analysis”, Duxbury Press, Pacific Grove, 1996

[12] Minhas U, Zhang J, Tran T, Cohen R., "Towards expanded trust management for agents in vehicular ad-hoc networks", International Journal of Computational Intelligence: Theory and Practice (IJCITP), vol. 5(1), pp. 3-15, 2010.

[13] Tajeddine, A., Kayssi, A., \& Chehab, A., "A Privacy-Preserving Trust Model for VANETs", 10th IEEE International Conference on Computer and Information Technology (CIT 2010), 2010, pp. 832-837.

[14] Huang, Z., Ruj, S., Cavenaghi, M., \& Nayak, A., " Limitations of Trust Management Schemes in VANET and Countermeasures" 2011 IEEE 22nd International Symposium on personal, indoor and mobile radio communications, 2011, pp. 1228-1232

[15] el mouna Zhioua, G., Tabbane, N., Labiod, H., \& Tabbane, S., “A Fuzzy Multi-Metric QoS-Balancing Gateway Selection Algorithm in a Clustered VANET to LTE Advanced Hybrid Cellular Network” IEEE Trans. on Vehicular Technology, vol. 64(2), pp. 804-817, 2015.

[16] Zhong, T., Xu, B., \& Wolfson, O., "Disseminating Real-Time Traffic Information in Vehicular Ad-Hoc Networks", 2008 IEEE Intelligent Vehicles Symposium, Jun 2008, pp. 1056-1061.

[17] Wang, X. B., Yang, Y. L., \& An, J. W. , "Multi-Metric Routing Decisions in VANET", 8th IEEE Int. Conf. on Dependable, Autonomic and Secure Computing, 2009, pp. 551-556.

[18] Yang, Y., Gao, Z., Qiu, X., Liu, Q., Hao, Y., \& Zheng, J., "A Hierarchical Reputation Evidence Decision System (REDS) in VANET", International Journal of Distributed Sensor Networks, 2015

[19] Fernandes, C. P., de Simas, I., de Mello, E. R., \& Wangham, M. S., "RS4VANETs - a decentralized reputation system for assessing the trustworthiness of nodes in vehicular networks," in 2015 International Wireless Communications and Mobile Computing Conference (IWCMC), pp.268-273, Aug. 2015

[20] Stoneburner G, Goguen A, Feringa A., "Risk management guide for information technology systems”, Tech. Rep. SP 800e30. NIST; July 2002.

[21] Shang, K., and Hossen, Z., "Applying Fuzzy Logic to Risk Assessment and Decision-Making." Research Report sponsored by Casualty Actuarial Society, Canadian Institute of Actuaries, 2013, Available at: http://www.soa.org/Files/Research/Projects/research-2013-fuzzylogic.pdf

[22] Miao, S., Hammell II, R. J., Hanratty, T., \& Tang, Z., “Comparison of Fuzzy Membership Functions for Value of Information Determination”, 
Proceedings of the 25th Modern Artificial Intelligence and Cognitive Science Conference, Spokane, Washington, USA, April 2014, pp. 53-60

[23] Young, K., Lee, J. D., \& Regan, M. A. (Eds.), "Driver distraction: Theory, effects, and mitigation” CRC Press, 2008

[24] McKnight, A. J., \& McKnight, A. S., "The effect of cellular phone use upon driver attention." Accident Analysis \& Prevention, vol. 25(3), pp. 259-265, 1993.

[25] Halleck, T., "Google Inc. Says Self-Driving Car Will Be Ready By 2020". International Business Times, 15 Jan 2015, Available at: http://www.ibtimes.com/google-inc-says-self-driving-car-will-be-ready2020-1784150

APPENDIX

TABLE I. DATA SET FOR RISK ESTIMATION

\begin{tabular}{|c|c|c|c|c|c|c|c|c|c|}
\hline \multirow{2}{*}{ \# } & \multirow{2}{*}{ 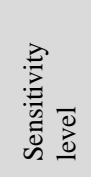 } & \multicolumn{6}{|c|}{ Vehicle context } & \multicolumn{2}{|c|}{ Driver's attitude } \\
\hline & & Lane & Weather & Time & Traffic & Road & Speed & Age & Experience \\
\hline 1 & 1 & Corner & Clear & Night & Car in rear & Icy & Decelerating & 45 & 40 \\
\hline 2 & 7 & Corner & Foggy & Day & Car on right & Wet & Decelerating & 14 & 42 \\
\hline 3 & 5 & Downhill & Raining & Night & Car on left & Icy & Accelerating & 20 & 47 \\
\hline 4 & 10 & Curve & Snowing & Night & Car in front & Icy & Accelerating & 65 & 38 \\
\hline 5 & 5 & Intersection & Snowing & Day & Car on right & Icy & Accelerating & 75 & 50 \\
\hline 6 & 5 & Corner & Snowing & Day & Car in front & Wet & Decelerating & 32 & 45 \\
\hline 7 & 8 & Curve & Clear & Day & Car on left & Dry & Decelerating & 53 & 47 \\
\hline 8 & 5 & Straight & Windy & Night & Car on left & Dry & Decelerating & 47 & 20 \\
\hline 9 & 2 & Corner & Foggy & Dusk & Car in rear & Dry & Accelerating & 24 & 50 \\
\hline 10 & 10 & Corner & Raining & Dawn & Car on left & Dry & Accelerating & 29 & 25 \\
\hline 11 & 10 & Straight & Windy & Dawn & Car in front & Dry & Decelerating & 22 & 47 \\
\hline 12 & 7 & Curve & Foggy & Dusk & Car in rear & Wet & Decelerating & 43 & 34 \\
\hline 13 & 7 & Intersection & Clear & Night & Car on left & Wet & Accelerating & 56 & 30 \\
\hline 14 & 7 & Straight & Snowing & Night & Car on right & Dry & Decelerating & 18 & 3 \\
\hline 15 & 5 & Intersection & Foggy & Dawn & Car in front & Icy & Accelerating & 76 & 13 \\
\hline 16 & 9 & Straight & Clear & Dawn & Car in rear & Dry & Accelerating & 14 & 30 \\
\hline 17 & 10 & Uphill & Clear & Dawn & Car on right & Icy & Accelerating & 38 & 34 \\
\hline 18 & 8 & Curve & Foggy & Day & Car in rear & Dry & Decelerating & 12 & 28 \\
\hline 19 & 9 & Downhill & Clear & Dawn & Car on left & Dry & Accelerating & 75 & 17 \\
\hline 20 & 2 & Corner & Foggy & Dawn & Car on right & Icy & Decelerating & 20 & 39 \\
\hline 21 & 1 & Corner & Windy & Dusk & Car on left & Wet & Decelerating & 37 & 50 \\
\hline 22 & 1 & Corner & Raining & Day & Car in front & Wet & Accelerating & 50 & 29 \\
\hline 23 & 10 & Corner & Windy & Night & Car on right & Icy & Decelerating & 67 & 14 \\
\hline 24 & 8 & Straight & Raining & Dawn & Car in front & Icy & Decelerating & 22 & 31 \\
\hline 25 & 9 & Intersection & Raining & Day & Car on right & Wet & Accelerating & 36 & 8 \\
\hline 26 & 1 & Corner & Windy & Dawn & Car on left & Dry & Accelerating & 79 & 1 \\
\hline 27 & 5 & Intersection & Clear & Night & Car on right & Dry & Decelerating & 20 & 21 \\
\hline 28 & 6 & Downhill & Snowing & Dusk & Car in front & Wet & Decelerating & 42 & 6 \\
\hline 29 & 10 & Corner & Raining & Dawn & Car on left & Dry & Accelerating & 24 & 41 \\
\hline 30 & 5 & Downhill & Snowing & Dusk & Car on left & Icy & Accelerating & 39 & 6 \\
\hline 31 & 1 & Corner & Clear & Night & Car in front & Wet & Decelerating & 55 & 50 \\
\hline 32 & 10 & Corner & Foggy & Day & Car on right & Icy & Accelerating & 36 & 1 \\
\hline 33 & 6 & Corner & Windy & Dawn & Car in rear & Icy & Decelerating & 16 & 42 \\
\hline 34 & 7 & Curve & Foggy & Dawn & Car in front & Dry & Decelerating & 17 & 14 \\
\hline 35 & 5 & Winding & Raining & Night & Car on left & Icy & Decelerating & 63 & 47 \\
\hline 36 & 10 & Curve & Clear & Dawn & Car on left & Wet & Accelerating & 65 & 39 \\
\hline 37 & 3 & Curve & Snowing & Dawn & Car in rear & Wet & Decelerating & 41 & 5 \\
\hline 38 & 7 & Straight & Foggy & Dawn & Car on right & Dry & Decelerating & 22 & 23 \\
\hline 39 & 9 & Corner & Clear & Dawn & Car on left & Icy & Accelerating & 49 & 5 \\
\hline 40 & 1 & Uphill & Windy & Dawn & Car in rear & Wet & Decelerating & 46 & 50 \\
\hline 41 & 8 & Curve & Snowing & Dawn & Car on right & Wet & Decelerating & 62 & 18 \\
\hline 42 & 6 & Downhill & Foggy & Dawn & Car in front & Dry & Decelerating & 50 & 32 \\
\hline 43 & 6 & Straight & Foggy & Night & Car on right & Icy & Decelerating & 40 & 12 \\
\hline 44 & 6 & Straight & Foggy & Dusk & Car on right & Icy & Decelerating & 59 & 48 \\
\hline 45 & 9 & Straight & Clear & Day & Car in rear & Icy & Decelerating & 28 & 19 \\
\hline 46 & 6 & Uphill & Clear & Night & Car in front & Wet & Decelerating & 68 & 7 \\
\hline 47 & 1 & Downhill & Clear & Day & Car on left & Icy & Decelerating & 26 & 41 \\
\hline 48 & 5 & Intersection & Foggy & Dawn & Car on right & Icy & Accelerating & 65 & 32 \\
\hline 49 & 7 & Uphill & Clear & Night & Car in front & Dry & Decelerating & 58 & 27 \\
\hline 50 & 3 & Uphill & Snowing & Dusk & Car on right & Icy & Accelerating & 18 & 36 \\
\hline 51 & 10 & Downhill & Windy & Dawn & Car on right & Dry & Decelerating & 12 & 40 \\
\hline 52 & 1 & Winding & Foggy & Dusk & Car on right & Dry & Accelerating & 13 & 29 \\
\hline
\end{tabular}




\begin{tabular}{|c|c|c|c|c|c|c|c|c|c|}
\hline 53 & 1 & Winding & Clear & Night & Car in rear & Dry & Accelerating & 14 & 8 \\
\hline 54 & 7 & Curve & Clear & Dawn & Car in rear & Dry & Decelerating & 60 & 15 \\
\hline 55 & 2 & Winding & Raining & Day & Car on right & Wet & Decelerating & 48 & 44 \\
\hline 56 & 10 & Straight & Foggy & Dusk & Car in front & Dry & Decelerating & 18 & 19 \\
\hline 57 & 6 & Corner & Raining & Dawn & Car on left & Icy & Accelerating & 40 & 9 \\
\hline 58 & 8 & Intersection & Foggy & Dawn & Car on right & Wet & Decelerating & 22 & 30 \\
\hline 59 & 6 & Corner & Raining & Day & Car in rear & Dry & Accelerating & 62 & 17 \\
\hline 60 & 9 & Uphill & Raining & Night & Car in rear & Icy & Decelerating & 31 & 18 \\
\hline 61 & 8 & Uphill & Windy & Day & Car in rear & Wet & Decelerating & 21 & 14 \\
\hline 62 & 2 & Intersection & Foggy & Dusk & Car on right & Dry & Accelerating & 17 & 33 \\
\hline 63 & 10 & Winding & Foggy & Night & Car in rear & Icy & Decelerating & 28 & 33 \\
\hline 64 & 1 & Downhill & Clear & Dawn & Car on left & Wet & Accelerating & 36 & 24 \\
\hline 65 & 6 & Winding & Clear & Dawn & Car in front & Icy & Decelerating & 13 & 27 \\
\hline 66 & 1 & Winding & Raining & Dawn & Car on left & Dry & Decelerating & 25 & 23 \\
\hline 67 & 2 & Downhill & Raining & Dusk & Car in rear & Icy & Accelerating & 54 & 17 \\
\hline 68 & 2 & Downhill & Clear & Day & Car on right & Wet & Accelerating & 25 & 45 \\
\hline 69 & 3 & Winding & Raining & Dawn & Car on left & Dry & Accelerating & 72 & 47 \\
\hline 70 & 10 & Winding & Snowing & Dusk & Car in front & Wet & Accelerating & 76 & 34 \\
\hline 71 & 2 & Intersection & Foggy & Dusk & Car in rear & Icy & Accelerating & 46 & 34 \\
\hline 72 & 1 & Corner & Snowing & Night & Car in front & Icy & Decelerating & 31 & 17 \\
\hline 73 & 7 & Curve & Clear & Day & Car on right & Dry & Accelerating & 22 & 5 \\
\hline 74 & 5 & Intersection & Windy & Dusk & Car in rear & Icy & Decelerating & 70 & 37 \\
\hline 75 & 8 & Uphill & Clear & Dusk & Car on left & Wet & Accelerating & 54 & 26 \\
\hline 76 & 7 & Downhill & Raining & Day & Car in front & Wet & Accelerating & 52 & 7 \\
\hline 77 & 2 & Intersection & Raining & Dusk & Car in rear & Icy & Decelerating & 47 & 44 \\
\hline 78 & 2 & Downhill & Clear & Dusk & Car on left & Dry & Decelerating & 49 & 28 \\
\hline 79 & 7 & Uphill & Snowing & Night & Car in front & Icy & Accelerating & 10 & 40 \\
\hline 80 & 9 & Intersection & Raining & Day & Car on left & Icy & Accelerating & 45 & 20 \\
\hline 81 & 1 & Corner & Raining & Day & Car on left & Icy & Accelerating & 47 & 5 \\
\hline 82 & 8 & Winding & Snowing & Dawn & Car in front & Dry & Decelerating & 47 & 48 \\
\hline 83 & 4 & Intersection & Foggy & Day & Car on right & Icy & Decelerating & 54 & 27 \\
\hline 84 & 2 & Corner & Clear & Night & Car on right & Wet & Decelerating & 19 & 46 \\
\hline 85 & 9 & Straight & Snowing & Dawn & Car in front & Icy & Decelerating & 71 & 42 \\
\hline 86 & 4 & Uphill & Snowing & Day & Car on right & Wet & Accelerating & 25 & 3 \\
\hline 87 & 1 & Downhill & Foggy & Dusk & Car in front & Dry & Decelerating & 10 & 27 \\
\hline 88 & 7 & Curve & Foggy & Dusk & Car on left & Icy & Accelerating & 46 & 21 \\
\hline 89 & 4 & Curve & Clear & Dawn & Car in front & Dry & Decelerating & 46 & 31 \\
\hline 90 & 7 & Corner & Snowing & Dusk & Car on right & Dry & Decelerating & 10 & 29 \\
\hline 91 & 9 & Curve & Windy & Dawn & Car on right & Dry & Accelerating & 67 & 20 \\
\hline 92 & 5 & Downhill & Foggy & Dawn & Car on right & Wet & Accelerating & 29 & 50 \\
\hline 93 & 8 & Winding & Raining & Dawn & Car on right & Wet & Accelerating & 10 & 18 \\
\hline 94 & 9 & Curve & Clear & Dawn & Car on right & Dry & Accelerating & 80 & 11 \\
\hline 95 & 10 & Intersection & Clear & Night & Car on left & Icy & Decelerating & 78 & 49 \\
\hline 96 & 4 & Straight & Snowing & Day & Car on left & Icy & Decelerating & 53 & 31 \\
\hline 97 & 1 & Curve & Foggy & Day & Car in rear & Wet & Accelerating & 69 & 41 \\
\hline 98 & 5 & Straight & Snowing & Dawn & Car in front & Wet & Decelerating & 57 & 37 \\
\hline 99 & 7 & Winding & Windy & Dawn & Car in front & Dry & Decelerating & 42 & 44 \\
\hline 100 & 10 & Downhill & Raining & Day & Car in rear & Wet & Accelerating & 67 & 5 \\
\hline
\end{tabular}

